\title{
On Nie-Tan Operator and Type-reduction of Interval Type-2 Fuzzy Sets
}

\author{
Jiawei Li, Member, IEEE, Robert John, Senior Member, IEEE, Simon Coupland, Member, IEEE, \\ and Graham Kendall, Senior Member, IEEE
}

\begin{abstract}
Type-reduction of type-2 fuzzy sets is considered to be a defuzzification bottleneck because of the computational complexity involved in the process of type-reduction. In this research, we prove that the closed-form Nie-Tan operator, which outputs the average of the upper and lower bounds of the footprint of uncertainty, is actually an accurate method for defuzzifing interval type-2 fuzzy sets.
\end{abstract}

Index Terms-Interval Type-2 fuzzy sets, type-reduction, defuzzification, Nie-Tan operator.

\section{INTRODUCTION}

$\mathbf{T}$ YPE-2 fuzzy sets (T2 FSs) generalize T1 FSs so that uncertainty associated with the membership function is taken into account [1]. Compared with a T1 FS, in which the membership is represented by crisp numbers, the membership of a T2 FS is represented by a FS, which is known as the secondary membership. Defuzzification is the final stage of a fuzzy inference system, in which a FS is converted into a crisp number. Unfortunately, defuzzification of a T2 FS can be so computationally complex that it has been known as the defuzzification bottleneck [2]. Defuzzification of T2 FSs usually contains two stages [3]: a type-reduction stage, in which the T2 FS is converted to a T1 FS, and a defuzzification of the T1 FS stage. It is the type-reduction stage that leads to the defuzzification bottleneck since there have been a number of efficient methods for defuzzification of T1 FSs. One of the most popular methods to defuzzify T1 FSs is the centroid $[4,5]$.

$$
d_{c}(\mu(x))=\frac{\int_{x_{\min }}^{x_{\max }} \mu(x) \cdot x d x}{\int_{x_{\min }}^{x_{\max }} \mu(x) d x}
$$

where $\mu(x)$ is the membership function of the T1 FS.

The difficulty in the extension of the centroid method to T2 defuzzification lies in the fact that, according to the representation theorem [6], a T2 FS is represented by the union

Manuscript received May 25, 2016; revised October 19, 2016; accepted January 18, 2017. This work was supported by the Engineering and Physical Sciences Research Council UK (EPSRC) grant reference EP/H000968/1 and grant reference $\mathrm{EP} / \mathrm{J} 017515 / 1$.

$\mathrm{J}$. Li is with Department of Computer Science and Mathematics, University of Stirling, Stirling, UK, FK9 4LA. e-mail: lij@cs.stir.ac.uk

R. John and G. Kendall are with School of Computer Science, University of Nottingham, Jubilee Campus, Wollaton Road, Nottingham, UK, NG8 1BB. e-mail: robert.john@ nottingham.ac.uk

S. Coupland is with School of Computer Science and Informatics, De Montfort University, The Gateway, Leicester, UK, LE1 9BH. e-mail: simon.coupland@gmail.com

G. Kendall is with School of Computer Science, University of Nottingham, Malaysia Campus, Jalan Broga, 43500 Semenyih, Selangor Darul Ehsan, Malaysia. e-mail: graham.kendall@ nottingham.edu.my of its embedded sets. The center of gravity (COG) of a T2 FS is the average of the centroids of all the embedded sets. An exhaustive type-reduction method [7] is usually impractical, depending on the scales of discretisation. The sampling method, in which a number of randomly selected samples are used to represent the whole union of embedded sets, is an efficient, cut-down alternative to the exhaustive method $[2,8,9]$. Some other general T2 defuzzification methods include: vertical slice centroid type-reduction (VSCTR)[10], plane presentation [11, 22], geometric method [24] and the collapsing method [12]. There is still not an accurate type-reduction of general T2 FSs other than the exhaustive algorithm.

Interval type-2 (IT2) FSs have received more attention in recent years because the mathematics that is needed for IT2 FSs is much simpler than the mathematics for general type-2 FSs[13-15]. The secondary memberships of IT2 FSs can be ignored because they are all set to one. Some operators have been proposed for IT2 defuzzification.

The Karnik-Mendel (KM) algorithm [16], which computes the smallest and largest centroid of embedded T1 FSs iteratively and then uses the average of two values as the result of defuzzification, is the most commonly used type-reduction method. A number of enhancements to KM algorithm have been made in order either to reduce the computational burden or to improve the accuracy [17-21].

Nie-Tan type-reduction operator has a simple closed-form representation [23]. To date, it is considered to be an approximation of KM algorithms, i.e. Nie-Tan operator is a firstorder Taylor series approximation of $\mathrm{KM}+$ defuzzification and an improved Nie-Tan operator is a third-order approximation of $\mathrm{KM}+$ defuzzification $[20,27]$. We will prove that NieTan operator is equivalent to exhaustive type-reduction and is actually an accurate type-reduction method for both concrete and continuous IT2 FSs in this paper.

This paper is organized as follows. Section 2 introduces some basic terms on IT2 FSs. Section 3 presents a theorem and the proof of it. Section 4 compares three type-reduction methods in defuzzifying four IT2 FSs. The paper is concluded in section 5 .

\section{BACKGROUND}

Let $X$ be a universe of discourse. A T1 FS on $X$ is characterised by a membership function $\mu_{A}: X \rightarrow[0,1]$, where the membership grades are crisp numbers. A T2 FS in $X$ is a fuzzy set whose membership grades are themselves fuzzy

$$
\mu_{\tilde{A}}: X \rightarrow[0,1]^{[0,1]}
$$


It can be expressed as

$$
\widetilde{A}=\left\{\left(x, \mu_{\widetilde{A}}(x)\right) \mid \mu_{\widetilde{A}}(x) \in \widetilde{P}(U), \forall x \in X\right\}
$$

where $X$ is called the primary domain and $U$ is the secondary domain and $\widetilde{P}(U)$ is the set of fuzzy sets in $U$ [2]. The membership function of a general T2 FS is three-dimensional, where the third dimension is called secondary membership, which may take any value from 0 to 1 . For an Interval T2 FS the secondary membership grades are all 1 . Some terms used in this paper are now defined.

The footprint of uncertainty (FOU) is the projection of the T2 FS onto the $x-u$ plane.

The upper and lower membership functions of a T2 FS are the T1 membership functions associated with the upper and lower bounds of the FOU respectively. The upper and lower membership functions are denoted by $\bar{\mu}(x)$ and $\mu(x)$ in this paper.

An embedded T1 set of a IT2 FS is a T1 FS whose membership function, $\mu(x)$, is within the FOU. For example, there must be $\mu(x) \leq \mu(x) \leq \bar{\mu}(x)$ at any $x$. Based on the concept of wavy slice [6], a IT2 FS can be considered as the union of all its embedded T1 sets.

A random embedded set is an embedded T1 FS whose membership function is randomly chosen, i.e. for each nonzero $\mathrm{x}$-value a random $\mu$-value $(\mu \leq \mu \leq \bar{\mu})$ is chosen.

A representative embedded set of an IT2 FS is a T1 FS, the centroid of which is equivalent to the COG of the IT2 FS [2]. Note that there might be unlimited number of representative embedded sets for an IT2 FS.

In Nie-Tan method, the average of upper and lower membership functions is computed as the membership function of a representative T1 embedded set. Let $\mu^{*}(x)$ be the membership function.

$$
\mu^{*}(x)=\frac{1}{2}(\bar{\mu}(x)+\underline{\mu}(x))
$$

The COG of $\widetilde{A}$ is equivalent to the centroid of $\mu^{*}(x)$. The advantages of Nie-Tan method include low computation cost and closed-form solutions [27].

\section{COG of AN IT2 FS}

The random sampling is an approximate method for IT2 type-reduction and defuzzification. It is intuitive to deduce that, if the sample size approaches infinity, a random sampling turns to be an accurate type-reduction and defuzzification method. We have the following theorems.

Theorem 1: A random sampling method computes the accurate COG of an IT2 FS when the number of sampling is infinity.

Proof: Let's first consider a discrete IT2 FS, $\tilde{A}$, defined in a universe of discourse, $X$. Let $M$ and $L$ denote the number of vertical slices along $x$-axis and number of horizontal slices along $\mu$-axis respectively. Since a T1 FS contains $M$ vertical slices, there are totally $L^{M}$ embedded T1 FSs in $\tilde{A}$.

Let $\bar{\mu}(x)$ and $\mu(x)$ denote the upper and lower memberships of FOU respectively. We randomly choose $N$ embedded T1 FSs of $\tilde{A}$. Let $\mu_{i}(x)$ be the membership function of $i^{\text {th }}$ embedded set, as shown in Fig. 1.
The aggregation of $N$ random embedded T1 FSs can be computed by

$$
\sum_{i=1}^{N} \mu_{i}(x)=\sum_{j=1}^{M} \sum_{i=1}^{N} \mu_{i}\left(x_{j}\right)
$$

Thus

$$
\lim _{N \rightarrow \infty} \frac{1}{N} \sum_{i=1}^{N} \mu_{i}(x)=\lim _{N \rightarrow \infty} \frac{1}{N} \sum_{i=1}^{N} \sum_{j=1}^{M} \mu_{i}\left(x_{j}\right)
$$

Because $\mu_{i}\left(x_{j}\right)$ is a random value uniformly distributed within $\left[\mu_{i}\left(x_{j}\right), \bar{\mu}_{i}\left(x_{j}\right)\right]$, the right side of above equation can be transformed to

$$
\lim _{N \rightarrow \infty} \frac{1}{N} \sum_{i=1}^{N} \sum_{j=1}^{M} \mu_{i}\left(x_{j}\right)=\frac{1}{L} \sum_{k=1}^{L} \sum_{j=1}^{M} \mu_{k}\left(x_{j}\right)
$$

Thus

$$
\left.\lim _{N \rightarrow \infty} \frac{L}{N} \sum_{i=1}^{N} \mu_{i}(x)=\sum_{k=1}^{L} \sum_{j=1}^{M} \mu_{k}\left(x_{j}\right)\right)
$$

The right side of the equation is exactly the aggregation of

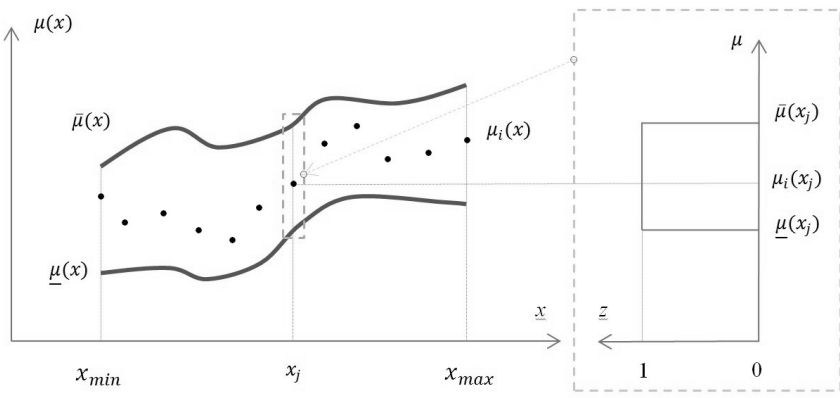

Fig. 1: Membership function of a random chosen embedded T1 FS, $\mu_{i}(x)$, depicted by the black dots, satisfies $\mu(x) \leq \mu_{i}(x) \leq$ $\bar{\mu}(x)$ at any $\mathrm{x}$. The secondary membership grade $\bar{a}$ any vertical slice $x_{j}$ is 1 .

membership functions of all embedded FSs of $\tilde{A}$ while the left side is a random sampling with $N \rightarrow \infty$. Note that $\frac{L}{N}$ in the left side of equation has no influence in computing the COG of $\tilde{A}$ because it is a constant. For example, if we replace $\mu_{i}(x)$ with $\mu_{i}^{\prime}(x)=\frac{L}{N} \mu_{i}(x)$ the centroid remains the same according to (1).

When $\tilde{A}$ is a continuous IT2 FS, we can simply replace $\sum_{j=1}^{M}$ and $\sum_{k=1}^{L}$ by $\int_{x_{\min }}^{x_{\max }}$ and $\int_{\mu}^{\bar{\mu}(x)}$ respectively and we have

$$
\lim _{N \rightarrow \infty} \frac{L}{N} \sum_{i=1}^{N} \mu_{i}(x)=\int_{x_{\min }}^{x_{\max }} \int_{\underline{\mu}(x)}^{\bar{\mu}(x)} \mu(x) d \mu d x
$$

Theorem 2: A representative $\mathrm{T} 1$ embedded set of $\tilde{A}$ has the membership function

$$
\mu^{*}(x)=\frac{1}{2}(\bar{\mu}(x)+\underline{\mu}(x))
$$

and the COG of $\tilde{A}$ can be computed by

$$
C(\tilde{A})=\frac{\int_{x_{\min }}^{x_{\max }} \mu^{*}(x) \cdot x d x}{\int_{x_{\min }}^{x_{\max }} \mu^{*}(x) d x}
$$


Proof: Suppose that we randomly choose $N$ embedded T1 FSs of a discretised IT2 FS $\tilde{A}$. Let $\mu_{i}(x)$ be the membership function of $i^{\text {th }}$ embedded set and $x_{j}$ be an arbitrary vertical slice, as shown in Fig. 1. $\mu_{i}\left(x_{j}\right)$ can be represented by $\mu_{i}\left(x_{j}\right)=\mu\left(x_{j}\right)+\omega_{i}\left(\bar{\mu}\left(x_{j}\right)-\mu\left(x_{j}\right)\right)$ where $\omega_{i}$ is an random number uniformly distributed within $[0,1]$. We have

$$
\begin{gathered}
\sum_{i=1}^{N} \mu_{i}\left(x_{j}\right)=\sum_{i=1}^{N}\left(\underline{\mu}\left(x_{j}\right)+\omega_{i}\left(\bar{\mu}\left(x_{j}\right)-\underline{\mu}\left(x_{j}\right)\right)\right) \\
=N \underline{\mu}\left(x_{j}\right)+\left(\bar{\mu}\left(x_{j}\right)-\underline{\mu}\left(x_{j}\right)\right) \sum_{i=1}^{N} \omega_{i}
\end{gathered}
$$

There must be

$$
\lim _{N \rightarrow \infty} \sum_{i=1}^{N} \omega_{i}=\frac{N}{2}
$$

because $\omega_{i}$ uniformly distributes within $[0,1]$. Then,

$$
\lim _{N \rightarrow \infty} \frac{1}{N} \sum_{i=1}^{N} \mu_{i}\left(x_{j}\right)=\frac{1}{2}\left(\bar{\mu}\left(x_{j}\right)+\underline{\mu}\left(x_{j}\right)\right)
$$

This equation holds for all $x$ since $x_{j}$ is an arbitrary vertical slice. Thus, we have

$$
\lim _{N \rightarrow \infty} \frac{1}{N} \sum_{i=1}^{N} \mu_{i}(x)=\frac{1}{2}(\bar{\mu}(x)+\underline{\mu}(x))
$$

According to Theorem 1 and (1), $\mu^{*}=\frac{1}{2}(\bar{\mu}(x)+\mu(x))$ is the membership function of a representative $\mathrm{T}^{2} \mathrm{FS}^{-}$and it computes the accurate COG of $\tilde{A}$.

It is easy to verify that the above proof applies to a continuous IT2 FS if we replace $\sum_{i=1}^{N}$ in the right side of (9) with $\int_{x_{\min }}^{x_{\max }}$.

Theorem 2 shows that Nie-Tan operator, in which the membership function of the output T1 FS is the average of upper and lower membership functions, is equivalent to an exhaustive type-reduction. Thus it computes the accurate COG of an IT2 FS.

\section{Numerical EXAMPLES}

In this section, we compare Nie-Tan operator with KM and the sampling methods on defuzzifying four IT2 FSs. Four examples of IT2 FSs are considered in a number of papers, such as [10-13]. The upper membership functions (UMF) and lower membership functions (LMF) of four IT2 FSs are defined by,

A. Symmetric Gaussian MFs with uncertainty deviation.

$$
\begin{array}{ll}
\underline{\mu}(x)=\exp \left(-0.5((x-5) / 0.25)^{2}\right) & x \in[0,10] \\
\bar{\mu}(x)=\exp \left(-0.5((x-5) / 1.75)^{2}\right) & x \in[0,10]
\end{array}
$$

$B$. Triangular LMF and Gaussian UMF.

$$
\begin{gathered}
\underline{\mu}(x)= \begin{cases}0.6(x+5) / 19 & \text { if } x \in[-5,2.6] \\
0.4(14-x) / 19 & \text { if } x \in[2.6,14]\end{cases} \\
\bar{\mu}(x)= \begin{cases}\exp \left(-0.5((x-2) / 5)^{2}\right) & \text { if } x \in[-5,7.185] \\
\exp \left(-0.5((x-9) / 1.75)^{2}\right) & \text { if } x \in[7.185,14]\end{cases}
\end{gathered}
$$

C. Piecewise Gaussian MFs.

$$
\begin{gathered}
\underline{\mu}(x)=\max \left\{0.5 \exp \left(-(x-3)^{2} / 2\right), 0.4 \exp \left(-(x-6)^{2} / 2\right)\right\} \\
x \in[0,10] \\
\bar{\mu}(x)=\max \left\{\exp \left(-(x-3)^{2} / 8\right), 0.8 \exp \left(-(x-6)^{2} / 8\right)\right\} \\
x \in[0,10]
\end{gathered}
$$

D. Piecewise Linear MFs.

$$
\begin{gathered}
\underline{\mu}(x)=\max \left\{\left[\begin{array}{ll}
(x-1) / 6 & \text { if } 1 \leq x \leq 4 \\
(7-x) / 6 & \text { if } 4<x \leq 7 \\
0 & \text { otherwise }
\end{array}\right],\right. \\
\left.\left[\begin{array}{ll}
(x-3) / 6 & \text { if } 3 \leq x \leq 5 \\
(8-x) / 9 & \text { if } 5<x \leq 8 \\
0 & \text { otherwise }
\end{array}\right]\right\} \\
\bar{\mu}(x)=\max \left\{\left[\begin{array}{ll}
(x-1) / 2 & \text { if } 1 \leq x \leq 3 \\
(7-x) / 4 & \text { if } 3<x \leq 7 \\
0 & \text { otherwise }
\end{array}\right],\right. \\
\left.\left[\begin{array}{ll}
(x-2) / 5 & \text { if } 2 \leq x \leq 6 \\
(16-2 x) / 5 & \text { if } 6<x \leq 8 \\
0 & \text { otherwise }
\end{array}\right]\right\}
\end{gathered}
$$

The accuracy of a sampling method depends on the number of samples. We adopt four random sampling methods which the number of samples are 20,50, 100 and 1,000 respectively. The scale of discetisation is set to be 0.01 . The results of defuzzification of four IT2 FSs are shown in Table. 1.

It shows that a random sampling method can be a close approximation of Nie-Tan operator, even with 20 samples. The $\mathrm{KM}$ method is not an accurate method although it provides the range of uncertainty involved in an IT2 FS. The KM method coincides with Nie-Tan operator when both UMF and LMF are symmetric, as in example $A$. However, KM method deviates from the accurate type reductions when either UMF or LMF is asymmetric.

\section{CONCLUSIONS AND FUTURE WORKS}

We have proved in two theorems that Nie-Tan operator computes accurate COGs of IT2 FSs. A closed-form typereduction operator has at least two advantages. First, it has less computational complexity than any numerical methods in defuzzifing continuous IT2 FSs. Second, it satisfies most of the desirable properties (so-called axioms) of type-1 defuzzification because of the closed-form nature, which could be important for a given application. For example, Nie-Tan operator satisfies the 12 properties of type- 2 defuzzification suggested in [25].

The method we used to prove theorem 2 has a close relationship with the sampling method. The error of a random sampling method tends to zero when the number of samples approaches infinity. Our future research is to apply this method to general T2 FSs type-reduction in order to generate a closedform operator.

\section{REFERENCES}

[1] Zadeh, L. A. (1975) The concept of a linguistic variable and its application to approximate reasoning-1, Information Sciences, 8(3): 199-249. 
TABLE I: Computation results.

\begin{tabular}{|c|c|c|c|c|c|c|c|}
\hline \multirow{2}{*}{ IT2 FS } & & \multicolumn{7}{|c|}{ Methods } \\
\cline { 3 - 8 } & & NT & S20 & S50 & S100 & S1000 & KM $^{*}$ \\
\hline \multirow{2}{*}{$A$} & COG & 5.0 & 5.0002 & 5.0005 & 5.0003 & 5.0001 & 5.0 \\
& Error & 0 & $0.02 \%$ & $0.05 \%$ & $0.03 \%$ & $0.01 \%$ & 0 \\
\hline \multirow{2}{*}{$B$} & COG & 3.714 & 3.7104 & 3.7138 & 3.7134 & 3.7135 & 3.7985 \\
& Error & 0 & $0.01 \%$ & $0.01 \%$ & $0.02 \%$ & $0.01 \%$ & $2.28 \%$ \\
\hline \multirow{2}{*}{$C$} & COG & 4.3952 & 4.394 & 4.3953 & 4.3951 & 4.395 & 4.4152 \\
& Error & 0 & $0.03 \%$ & $<0.01 \%$ & $<0.01 \%$ & $<0.01 \%$ & $0.46 \%$ \\
\hline \multirow{2}{*}{$D$} & COG & 4.3208 & 4.3198 & 4.3208 & 4.3206 & 4.3207 & 4.3261 \\
& Error & 0 & $0.02 \%$ & $<0.01 \%$ & $<0.01 \%$ & $<0.01 \%$ & $0.12 \%$ \\
\hline
\end{tabular}

[2] Greenfield, S. (2012) Type-2 fuzzy logic: circumventing the defuzzification Bottleneck, PhD Thesis, De Montfort University.

[3] Mendel, J. M. (2001) Uncertain rule-based fuzzy logic system: introduction and new directions. Prentice-Hall PTR.

[4] Runkler, T. A. (1997) Selection of appropriate defuzzification methods using application specific properties. IEEE Transactions on Fuzzy Systems, 5(1): 72-79.

[5] Fortemps, P., and Roubens, M. (1996) Ranking and defuzzification methods based on area compensation. Fuzzy sets and systems, 82(3): 319-330.

[6] Mendel, J. and John, R. (2002) Type-2 fuzzy sets made simple. IEEE Transactions on Fuzzy Systems, 10(2): 117-127.

[7] Greenfield, S., Chiclana, F., and John, R. (2009) Type-reduction of the discretised interval type-2 fuzzy set. In Proceedings of FUZZ-IEEE 2009, Jeju Island, Korea, pp. 738-743.

[8] Greenfield, S., John, R., and Coupland, S. (2005) A novel sampling method for type-2 defuzzification. In Proc. UKCI 2005, London, pp. 120127.

[9] Greenfield, S., Chiclana, F., John, R., and Coupland, S. (2012) The sampling method of defuzzification for type-2 fuzzy sets: experimental evaluation Information Sciences, 189: 77-92.

[10] John, R. (2000). Perception modelling using type-2 fuzzy sets. PhD Thesis, De Montfort University.

[11] Liu, F. (2008) An efficient centroid type-reduction strategy for general type-2 fuzzy logic system, Information Sciences, 178(9): 2224-2236.

[12] Greenfield, S., Chiclana, F., Coupland, S., and John, R. (2009) The collapsing method of defuzzification for discretised interval type-2 fuzzy sets. Information Sciences, 179(13): 2055-2069.

[13] Mendel, J., John, R. and Liu, F. (2006) Interval Type-2 Fuzzy Logic Systems Made Simple, IEEE Transactions on Fuzzy Systems, 14(6): 808821.

[14] Mendel, J., Liu, F. and Zhai, D. (2009) -plane representation for type2 fuzzy sets: Theory and applications, IEEE Transactions on Fuzzy Systems, 17(5): 1189-1207.

[15] Liang, Q. and Mendel, J. (2000). Interval type-2 fuzzy logic systems: theory and design. IEEE Transactions on Fuzzy Systems, 8(5): 535-550.

[16] Karnik, N. and Mendel, J. (2001) Centroid of a type-2 fuzzy set. Information Sciences, 132:195-220.

[17] Wu, D. and Mendel, J. (2009). Enhanced karnik-mendel algorithms. IEEE Transactions on Fuzzy Systems, 17(4): 923-934.

[18] Mendel, J. and Liu, F. (2007) Super-exponential convergence of the Karnik-Mendel algorithms for computing the centroid of an interval type2 fuzzy set, IEEE Transactions on Fuzzy Systems, 15(2): 309-320.

[19] Melgarejo, M. (2007) A fast recursive method to compute the generalized centroid of an interval type-2 fuzzy set. In Fuzzy Information Processing Society, 2007. NAFIPS'07. IEEE Annual Meeting of the North American, pp. 190-194.

[20] Mendel, J. and Liu, X. (2012) New closed form solutions for KarnikMendel algorithm +defuzzification of an interval type-2 fuzzy set, IEEE World Congress on Computational Intelligence, Australia, pp. 1-8.

[21] Liu, X., Mendel, J., and Wu, D. (2012) Study on enhanced karnikmendel algorithms: Initialization explanations and computation improvements, Information Sciences, 184(1): 75-91.

[22] Wagner, C. and Hagras, H. (2008) z slices-towards bridging the gap between interval and general type-2 fuzzy logic, in Proc. IEEE FUZZ Conference, Hong Kong, China, pp. 489-497.
[23] Nie, M. and Tan, W. (2008) Towards an efficient type-reduction method for interval type- 2 fuzzy logic systems, in Proc. IEEE Int. Conf. Fuzzy Syst.,pp. 1425-1432.

[24] Coupland, S. and John, R. (2007) Geometric type-1 and type-2 fuzzy logic systems, IEEE Transactions on Fuzzy Systems, 15(1): 3-15.

[25] Runkler, T., Coupland, S. and John, R. Properties of interval type-2 defuzzification operators, manuscript submitted to IEEE Transactions on Fuzzy Systems.

[26] Bezdek, J. (1993) Fuzzy models-what are they, and why? IEEE Transactions on Fuzzy Systems, 1(1): 1-6.

[27] Mendel, J. and Liu, X. (2013) Simplified Interval Type-2 Fuzzy Logic Systems, IEEE Transactions on Fuzzy Systems, 21(6): 1056-1069. 